Gaiser, Johanna M.; Kielblock, Stephan

\title{
Von Hausaufgaben zu rhythmisierten Lernzeiten. Ganztagsschulentwicklung am Beispiel einer längsschnittlichen Fallstudie
}

formal und inhaltlich überarbeitete Version der Originalveröffentlichung in:

formally and content revised edition of the original source in:

Zeitschrift für Bildungsforschung 9 (2019) 2, S. 159-175

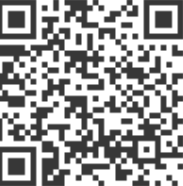

Bitte verwenden Sie in der Quellenangabe folgende URN oder DOI /

Please use the following URN or DOI for reference:

urn:nbn:de:0111-pedocs-181684

$10.25656 / 01: 18168$

https://nbn-resolving.org/urn:nbn:de:0111-pedocs-181684

https://doi.org/10.25656/01:18168

\section{Nutzungsbedingungen}

Gewährt wird ein nicht exklusives, nicht übertragbares, persönliches und beschränktes Recht auf Nutzung dieses Dokuments. Dieses Dokument ist ausschließlich für den persönlichen, nicht-kommerziellen Gebrauch bestimmt. Die Nutzung stellt keine Übertragung des Eigentumsrechts an diesem Dokument dar und gilt vorbehaltlich der folgenden Einschränkungen Auf sämtlichen Kopien dieses Dokuments müssen alle Urheberrechtshinweise und sonstigen Hinweise auf gesetzlichen Schutz beibehalten werden. Sie dürfen dieses Dokument nicht in irgendeiner Weise abändern, noch dürfen Sie dieses Dokument für öffentliche oder kommerzielle Zwecke vervielfältigen, öffentlich ausstellen, aufführen, vertreiben oder anderweitig nutzen.

Mit der Verwendung dieses Dokuments erkennen Sie die Nutzungsbedingungen an.

\section{Terms of use}

We grant a non-exclusive, non-transferable, individual and limited right to using this document.

This document is solely intended for your personal, non-commercial use. Use of this document does not include any transfer of property rights and it is conditional to the following limitations: All of the copies of this documents must retain all copyright information and other information regarding legal protection. You are not allowed to alter this document in any way, to copy it for public or commercial purposes, to exhibit the document in public, to perform, distribute or otherwise use the document in public.

By using this particular document, you accept the above-stated conditions of use.

\section{Kontakt / Contact:}

\section{peDOCS}

DIPF | Leibniz-Institut für Bildungsforschung und Bildungsinformation Informationszentrum (IZ) Bildung

E-Mail: pedocs@dipf.de

Internet: www.pedocs.de

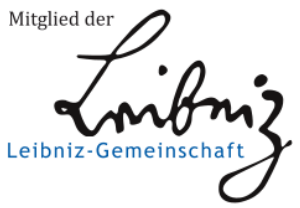


Johanna M. Gaiser und Stephan Kielblock

Von Hausaufgaben zu rhythmisierten Lernzeiten.

Ganztagsschulentwicklung am Beispiel einer längsschnittlichen Fallstudie

\section{Johanna M. Gaiser}

Institut für Erziehungswissenschaft

Justus-Liebig-Universität Gießen

Karl-Glöckner-Str. 21B

D-35394 Gießen

ORCID: 0000-0003-2882-4872

Dr. Stephan Kielblock

DIPF | Leibniz-Institut für Bildungsforschung und Bildungsinformation

Rostocker Straße 6

D-60323 Frankfurt am Main

ORCID: 0000-0003-3853-8554

Korrespondierende Autorin: Johanna M. Gaiser

johanna.m.gaiser@ew.jlug.de

+496419924074 


\title{
Von Hausaufgaben zu rhythmisierten Lernzeiten. Ganztagsschulentwicklung am Beispiel einer längs-
} schnittlichen Fallstudie

Zusammenfassung An deutschen Ganztagsschulen werden Hausaufgaben zunehmend durch individualisierte Lern- und Übungszeiten ersetzt. Mit verschiedenen Argumenten wird dieser Wandel in der schulischen Lernkultur begründet. Unter anderem gehen damit Vorteile für Schüler/innen und Eltern einher - Schüler/innen haben beispielsweise im Anschluss an den Schultag nicht zusätzliche Aufgaben für den Unterricht zu erledigen, Eltern haben nicht mehr dafür Sorge zu tragen, dass ihre Kinder diese Aufgaben auch erledigen. Für die Ganztagsschulen gehen mit einer Neustrukturierung der Aufgabenpraktiken jedoch entsprechende Herausforderungen einher. Dieser Beitrag geht der Frage nach, wie sich die Implementation einer voll integrierten Lernzeit an einer Schule vollzieht, an der es zuvor eine Hausaufgabenbetreuung gab. Hierzu wird die Fallstudie einer integrierten Gesamtschule vorgelegt, die anhand von längsschnittlichen qualitativen Daten rekonstruiert, welche Prozesse mit der Einführung einer neuen Aufgabenpraktik vor Ort ablaufen. Als Ergebnis zeichnen sich drei Ebenen ab, auf welchen es im Zuge der Einführung der Lern- und Übungszeit Veränderungen zu beobachten gibt: Zeitstruktur, Personaleinsatz und Lernbegleitung.

Schlüsselwörter Hausaufgaben $\cdot$ Lernzeit $\cdot$ Ganztagsschule $\cdot$ Fallstudie $\cdot$ Schulentwicklung

From homework to learning time. All-day school development using the example of a longitudinal case study

\begin{abstract}
At many German all-day schools, homework practices are being replaced by time at school for individualised learning, so-called learning time. A variety of arguments support this change in learning culture at school. Benefits are that pupils have almost no tasks to do after school and, accordingly, parents are no longer responsible for their children's homework. However, the schools have to handle the challenges of restructuring the learning practices. This study focuses on the question, which aspects of the schools' organisation are affected by the implementation of individualised learning time. Based on qualitative, longitudinal data of a German comprehensive school, different processes of organisational change are described in a case study. As a result, three layers are identified, which show significant changes after the implementation of learning time: time structure, personnel and learning support.
\end{abstract}

Keywords Homework $\cdot$ Learning time $\cdot$ All-day school $\cdot$ Case study $\cdot$ School development 


\section{Hausaufgaben und Lernzeiten an der Ganztagsschule}

Durch die zusätzliche Zeit, welche Schüler/innen in Ganztagsschulen verbringen, ergeben sich Veränderungen für die bisherigen Hausaufgabenpraktiken. Die vormals zu Hause und nach der Schule zu erledigenden Aufgaben werden zunehmend in die Schule verlagert. Begründet wird die Verlagerung der Hausaufgaben in den Schulalltag unterschiedlich. Neben einer zeitlichen Argumentation, dass die Schüler/innen nach einem verlängerten Schultag durch das Erledigen der Hausaufgaben zusätzlich belastet seien (Rabenstein und Podubrin 2015, S. 219), findet sich im Ganztagsschuldiskurs auch das Argument, dass die Integration der Hausaufgaben in den Schultag zur Chancengleichheit beitrage (Dollinger 2014, S. 79). Insbesondere Kinder, die zu Hause bei der Erledigung ihrer Aufgaben nicht unterstützt werden, können demnach von einem schulischen Angebot profitieren (ebd.). Dies fordert das konventionelle und allgemein gebräuchliche Verständnis von Hausaufgaben, als Aufgaben, die zu Hause zu erledigen sind, heraus.

Von den Bundesländern in Deutschland wird die Einführung von Hausaufgabenbetreuung bzw. von Übungs- und Lernzeiten betont. Beispielsweise wird in der aktuellen Fassung der Richtlinie für ganztägig arbeitende Schulen in Hessen (Hessisches Kultusministerium, 2018, Abschnitt 2) festgehalten, dass die Bereitstellung einer „(Haus)Aufgabenhilfe und -betreuung oder eine angeleitete Übungs- und Lernzeit" ein fester Bestandteil von Ganztagsschulen zu sein hat. In der hier gewählten Formulierung „(Haus-)Aufgaben“ deutet sich an, dass sich die Logik der zu Hause zu erledigenden Aufgaben - insbesondere in der gebundenen Form der Ganztagsschule - zunehmend auflöst zugunsten von Aufgaben, die in der Schule zu erledigen sind. Ähnliche Forderungen, insbesondere an Ganztagsschulen die Hausaufgaben in den Schultag zu integrieren, finden sich auch in anderen landespolitischen Dokumenten (u. a. Schulgesetz Berlin, Stand 2016).

Hausaufgaben sind in einem klassischen Sinne solche Aufgaben, deren Zielsetzung auf den Unterricht bezogen ist, zugleich aber in einem nicht-unterrichtlichen Setting stattfinden - mit anderen Worten: die Aufgaben werden neben dem curricularen Unterricht aber zugleich für den Unterricht erledigt (vgl. hierzu Gaiser et al. 2016; Höhmann 2005; Haenisch 2009).

Unter Lernzeiten (teilweise auch: ,Übungszeiten') sind Lern- und Übungsphasen für die Bearbeitung von Aufgaben gemeint, die zwar innerhalb der Schule, jedoch nicht im Unterricht stattfinden (Grimm und Schulz-Gade 2015, S. 36). Die Autor/innen beschreiben dies als sog. teilintegratives Modell der Hausaufgaben bzw. Lernzeiten. Im Gegensatz hierzu wäre im sog. integrativen Modell das Lernen und Üben gänzlich in den Unterricht integriert (ebd., S. 37). Im vorliegenden Beitrag und der dazugehörigen Einzelfallstudie werden die Lernzeiten des teilintegrativen Modells genauer in den Blick genommen.

Hascher und Bischof (2000) unterscheiden zwischen integrierten Hausaufgaben, die in der Schule erledigt werden, und traditionellen Hausaufgaben, die klassisch zu Hause erledigt werden. Die Ergebnisse ihrer Studie zeigen, dass die Mathematikleistung sich nicht unterscheidet zwischen der einen und der anderen Hausaufgabenvariante; dass aber bei denjenigen, die die Hausaufgaben in der Schule (integriert) erledigten, der Lernstress zu Hause signifikant geringer und die Einstellungen zur Schule signifikant höher waren (ebd.). Im Gegensatz dazu bieten Wild und Lorenz (2010) einen kritischen Blick auf die Integration der Hausaufgaben in die Schule. Sie führen an, dass sich die Eltern zwar dadurch entlastet fühlen könnten, somit allerdings auch deutlich weniger über die „schulischen Belange ihrer Kinder" informiert seien (ebd., S. 58).

Lern- und Übungszeiten haben sich nach wie vor noch nicht umfassend gegen die Hausaufgaben im althergebrachten Sinn durchgesetzt (Emden 2016, S. 195). Bei der Frage wie sich Hausaufgaben sinnvoll in die Ganztagsschule integrieren lassen, sind viele Schulen noch am Anfang ihrer Entwicklung (Grimm und Schulz-Gade 2015, S. 11). Aus diesen Entwicklungsbemühungen gehen verschiedene ,[n]eue Organisationsformen, die von der Hausaufgabenhilfe bis zu individuellen Lernzeiten mit einer neuen methodisch-didaktischen Qualität reichen“, hervor (ebd.). Praxisberichte verdeutlichen umfassend, mit welcher Komplexität es die Beteiligten bei der Implementation einer neuen Hausaufgabenpraxis zu tun haben (vgl. ebd.; Voag 2006). Der Einbezug der Lehrkräfte, Schüler/innen, Mitarbeiter des Ganztagsbereichs sowie der Eltern hängen mit einer erfolgreichen Realisierung veränderter Aufgabenpraktiken zusammen (Standop 2013, S. 310f.). Veränderungen im Bereich der Hausaufgabenbetreuung bzw. der Lern- und Übungszeiten wirken sich auch auf andere Bereiche der Schule aus - nicht zuletzt auch auf die Kooperation zwischen den pädagogisch Tätigen (ebd., S. 104).

Damit stellt sich die Frage, wie solche Aufgaben, die nunmehr in der Schule zu erledigen sind, in den schulischen Alltag implementiert werden können. Konkret wird in dem vorliegenden Beitrag die Fragestellung verfolgt, wie sich die Implementation von einer konventionellen Hausaufgabenpraxis (Hausaufgaben + Hausaufgabenbetreu- 
ung) zu einer voll integrierten Lernzeit vollzieht. Untersucht wird anhand der Einzelfallstudie zu welchen Veränderungen es auf unterschiedlichen Ebenen der Implementation kommt und wo im Laufe der ersten Schulhalbjahre nach Einführung der Lernzeit nachgesteuert werden muss.

Für die Schul- und Ganztagsschulforschung ist diese Fragestellung von Bedeutung, um die ablaufenden Prozesse bei der Implementation von Hausaufgaben- und Lernangeboten als einen Teil der Ganztagsschulentwicklung besser zu verstehen. Von ersten Hinweisen auf Gelingens- und Misslingensbedingungen für die Implementation könnte insbesondere auch die Ganztagsschulpraxis profitieren. Die Beantwortung dieser Fragestellung würde Hinweise dahingehend liefern, welche organisatorischen Herausforderungen eine solche Veränderung mit sich bringen kann.

\section{Forschungsstand}

Der außerunterrichtliche Bereich kann in Ganztagsschulen vergleichsweise flexibel gestaltet werden. Der einfachste Modus dürfte sein, dass im Unterricht Hausaufgaben aufgegeben werden, und dass die Ganztagsschule zudem beispielsweise nachmittags eine Hausaufgabenbetreuung anbietet, in der die Schüler/innen die Hausaufgaben unter Aufsicht bzw. Betreuung erledigen können. Komplexer wird es, wenn die Hausaufgaben in neuere Formen des betreuten Lernens, wie z. B. der Wochenplanarbeit (Meyer-Hamme 2014, S. 142) einmünden sollen, welche individueller auf die Schüler/innen zugeschnitten sind. Hier sind organisatorische Umstellungen nötig, um für solche Lernzeiten ein Zeitfenster zu finden; und es sind konzeptionelle Entwicklungen nötig, um das Potenzial des Außerunterrichtlichen (vgl. Kielblock 2015; StEG-Konsortium 2016) nicht unausgeschöpft zu lassen. Dem Anspruch einer individuellen Förderung können Ganztagsschulen bisher noch nicht immer gerecht werden. Heyl et al. (2016) führen das u. a. darauf zurück, dass für die Durchführung der Hausaufgabenbetreuung nicht immer geeignetes Fachpersonal bereitgestellt werde. Der Bildungsaspekt scheint gegenüber einer verlässlichen Betreuung häufig in den Hintergrund zu geraten (ebd., S. 183).

Tatsächlich ist eine Hausaufgabenbetreuung an der Schule anzubieten, relativ weit verbreitet. Knapp $90 \%$ der Ganztagsschulen im Primar- und Gymnasialbereich sowie etwa $75 \%$ im nicht-gymnasialen Sekundarstufen-IBereich geben an, Hausaufgabenbetreuung anzubieten (StEG Konsortium 2015, S. 74). Ganztagsschulen schaffen es aber häufig nicht, Hausaufgaben gänzlich in die Schule zu verlegen. In einer Befragung in Nordrhein-Westfalen (NRW) gibt mehr als die Hälfte der Eltern von Ganztagsschüler/innen im Schuljahr 2013/14 an, dass ihre Kinder mindestens ein Mal pro Woche auch zu Hause noch Hausaufgaben zu erledigen haben (Börner et al. 2014, S. 48). Nordt und Röhner (2008) zeigen: Der Zeitpunkt des Erledigens der Hausaufgaben ist häufig nicht frei wählbar, was den Schüler/innen missfällt. Ein weiterer Befund aus NRW besagt, dass Lernzeiten und Hausaufgabenbetreuung zunehmend auch während der Mittagszeit angeboten werden (Börner et al. 2013, S. 12).

Auf ganz Deutschland bezogen lässt sich feststellen, dass im ganztagsschulischen Primarbereich die Hausaufgabenbetreuung überwiegend von Personen des weiteren pädagogisch tätigen Personals durchgeführt wird; im Sekundarbereich ist der Anteil der Schulen geringer, der angibt, dass Hausaufgabenhilfe überwiegend bzw. ausschließlich vom weiteren pädagogisch tätigen Personal durchgeführt wird (Dieckmann et al. 2008, S. 176-177; vgl. auch Rollett und Tillmann 2009). Es scheint sich als günstig zu erweisen, wenn die Lehrkräfte selbst die Hausaufgabenbetreuung übernehmen, denn so können sie im Dialog mit den Kindern über diese zusätzlichen Aufgaben bleiben, die Aufgabenstellung ggf. anpassen (Haenisch 2009, S. 21) und im Gegensatz zu den traditionellen Hausaufgaben direktes Feedback zu den Aufgaben geben (Emden 2016, S. 196).

Das Setting in (Haus-)Aufgabenangeboten unterscheidet sich oftmals kaum vom Unterricht. Bundesweit befragte Schüler/innen geben an, dass sich die Hausaufgabenbetreuung im Hinblick auf die effektive Zeitnutzung und das Unterstützungsverhalten der Angebotsleitung kaum vom Fachunterricht unterscheidet (Radisch et al. 2008). Es wurde der Frage nachgegangen, durch welche pädagogischen Praktiken der pädagogisch Tätigen das Arbeiten der Schüler/innen „strukturiert, begleitet und kommentiert und unterstützt wird“ (Rabenstein und Podubrin 2015, S. 234). Das Autorenteam zieht das Fazit: In Hausaufgabensettings vollzieht sich „ein routinierter, auf schnelle Erledigung setzender Umgang mit Hausaufgaben“ (ebd., S. 242). Die pädagogischen Praktiken sind davon dominiert, „die Zeit effektiv zu nutzen“ und die Schüler/innen „im Modus permanenter konzentrierter Aktivität zu halten" (ebd., S. 243). Zudem zeigt eine andere Untersuchung, dass die Schüler/innen die außerunterrichtlichen Angebote jedoch auch in Abgrenzung zum Unterricht sehen und gerade die anderen Möglichkeiten zu lernen, die sie in den Angeboten sehen, wertschätzen (StEG Konsortium 2016).

Aus der Perspektive der Kinder kommen den pädagogisch Tätigen laut Nordt und Röhner (2008) zwei Aufgaben zu: zum einen die Unterstützung bei den Aufgaben, welche die Schüler/innen nicht ohne Hilfe lösen können, und 
zum anderen die Kontrolle der bearbeiteten Hausaufgaben (ebd., S. 75). Die Autorinnen zeigen: Die Hausaufgabenbetreuung wird stark strukturiert und als geregelter Raum wahrgenommen. Dies - insbesondere leise sein zu müssen - empfinden die Schüler/innen als negativ (ebd., S. 73-74). Weiter formulieren die Autorinnen folgende Kritik: „Der Typus des Lernens, der von den Kindern beschrieben wird, ist reproduktiv und das Erledigen von fremdbestimmten Aufgaben in einem stark reglementierten sozialen Kontext steht im Vordergrund“ (ebd., S. 76). Hausaufgabensettings scheinen sich insbesondere durch fehlende Freiheitsspielräume auszuzeichnen, sodass von Seiten der Kinder berichtet wird, dass uninteressante Aufgaben zu Langeweile führen und dass die Aufgaben zu viel und zu schwer sind. Sich als Schüler/in in einem solchen Setting als kompetent zu erleben, erscheint nicht leicht (Nordt 2013). Auch eine nicht ausreichende Zusammenarbeit zwischen den Lehrkräften, die die Aufgaben erteilen, und ihren Kooperationspartnern, welche die Hausaufgabenbetreuung anbieten, kann zu Schwierigkeiten in der erfolgreichen Umsetzung der Lernsettings führen (vgl. Gaiser et al. 2016).

Nicht zuletzt um von dieser von Reglementierung und Kontrolle geprägten Hausaufgabenkultur Abstand zu nehmen und den Schüler/innen mehr Selbstbestimmung zuzugestehen, wird an Ganztagsschulen zunehmend auf Aufgaben im ursprünglichen Sinn verzichtet. Lern- und Übungszeiten, also Phasen, in denen die Kinder ihre Aufgaben je nach Lernstand und -tempo eigenständig auswählen, bearbeiten und auf Richtigkeit überprüfen, werden erprobt. In diesem Zuge wird auch die Arbeit mit Wochenplänen aufgegriffen. Die Schüler/innen übernehmen hier selbst Verantwortung dafür, wie sie sich die Aufgaben im Tages- bzw. Wochenverlauf einteilen (Kaufmann 2013, S. 7). Insbesondere die den Hausaufgaben zugesprochenen Funktionen im erzieherischen Bereich werden in der Wochenplanarbeit umgesetzt: die Unterstützung bei der Entwicklung von Selbständigkeit, das Wecken von Arbeitsfreude und Interesse, der Erwerb von Organisationsfähigkeiten, das Stärken des Selbstvertrauens und das Bewirken einer positiven Grundhaltung gegenüber dem selbständigen Arbeiten (Emden 2016, S. 192; vgl. hierzu auch Heyl et al. 2018). Um auch den Funktionen im didaktisch-methodischen Bereich gerecht zu werden, müssen Lernund Übungszeiten und die Arbeit mit Wochenplänen so organisiert sein, dass die Aufgaben inhaltlich tatsächlich an den Unterricht anschließen und ggf. in diesem auch wieder thematisiert werden können (vgl. Standop 2013, S. 11f.).

Die Forschungsbefunde zusammenfassend kann festgestellt werden, dass Hausaufgaben an Ganztagsschulen vielfach noch als klassische Hausaufgaben konzeptioniert sind, die mit einer Hausaufgabenbetreuung im Ganztag flankiert wird. In diesem Fall kann es sein, dass die Schüler/innen trotzdem zu Hause noch Aufgaben erledigen müssen, dass vor allem weiteres Personal mit der Betreuung der Hausaufgaben betraut wird, und dass es in den Hausaufgabenbetreuungszeiten um das Erledigen der Hausaufgaben geht und nicht um das Lernen und die Beschäftigung mit Lerninhalten. Keine der vorliegenden Studien eröffnet einen Blick auf die Komplexität und das Zusammenwirken der Facetten, die es zu beachten gilt, wenn Hausaufgaben zunehmend in den Ganztag implementiert werden sollen. Zudem scheint es an längsschnittlichen Betrachtungen zu fehlen, welche die organisationalen Dynamiken beschreiben, die durch die Implementation der Hausaufgaben als Lernzeiten in den Ganztag auftreten. Der vorliegende Beitrag macht einen ersten Vorstoß, diese Forschungslücke zu schließen, indem eine Fallstudie zu einer Ganztagsschule vorgelegt wird.

\section{Methode}

Die Bearbeitung der Fragestellung erfordert einerseits eine längsschnittliche Datenlage, anhand derer die einzelnen Schritte der Implementation im Zeitverlauf analysiert werden können. Andererseits beinhaltet die Fragestellung zudem aber auch verschiedene Perspektiven. So macht es ggf. einen Unterschied, wie die Schulleitung oder die Angebotsleitung eine Neuerung (pädagogisch) intendiert hat und wie die Schüler/innen diese Neuerung nun tatsächlich wahrnehmen und nutzen.

\subsection{Hintergrund der Studie und empirisches Material}

Im Rahmen des Gießener Teilprojekts der Studie zur Entwicklung von Ganztagsschulen (StEG-Q; 2012-2015; BMBF gefördert) wurde eine Integrierte Gesamtschule, im Folgenden Pestalozzischule ${ }^{1}$ genannt, über einen Zeitraum von zwei Jahren untersucht. In dieser Zeit wurde die konventionelle Hausaufgabenpraxis (Hausaufgaben + Hausaufgabenbetreuung) in die Arbeit mit Wochenaufgaben überführt. Der gebundene Ganztagsbetrieb startete an der Pestalozzischule beginnend im Schuljahr 2011/12. Parallel dazu wurde die Hausaufgabenbetreuung im Ganztag für alle Schüler/innen eingeführt. Im Schuljahr 2013/14 wurde dann eine neue Art der Aufgabenbearbeitung in der Schule implementiert. Dieser Wandel in Teilschritten ist für die hier untersuchte Implementation einer neuen, selbstbestimmten Hausaufgabenpraxis von Bedeutung.

\footnotetext{
${ }^{1}$ Name von den Autor/innen geändert.
} 
Im dritten und vierten Schuljahr mit ganztägigem Betrieb wurden von StEG-Q an der Schule Datenerhebungen durchgeführt (2013/14 Feldphase $1^{2}$ und Feldphase 2 sowie 2014/15 Feldphase 3). Das Projekt interessierte sich vordergründig für außerunterrichtliche Angebote. Entsprechend dieses Fokus wurde mit den Schulleitungen abgestimmt, welche Personen über die Angebote potenziell Auskunft geben können (bspw. Kinder, die auch tatsächlich Angebote besuchen bzw. besucht haben). Über die Schulleitungen wurden diese Lehrkräfte und Personen des weiteren Personals sowie Schüler/innen hinsichtlich der Studie schriftlich informiert und zur Teilnahme eingeladen. Erwachsene konnten schriftlich einwilligen teilzunehmen. Auch die Schüler/innen konnten schriftlich einwilligen, wurden dann aber nur in die Studie einbezogen, wenn auch ihre Eltern der Teilnahme zustimmten. Die Angebote, um die es in den Interviews und Gruppendiskussionen ging, wurden durch die Projektmitarbeitenden besucht und teilnehmend beobachtet.

Für die vorliegende Studie wurden ausschließlich diejenigen Personen ausgewählt, die über die frühere Hausaufgabenbetreuung und/oder die neu eingeführte, selbstbestimmte Aufgabenpraxis Auskunft gegeben haben. Durch dieses Auswahlverfahren ergibt sich für die vorliegende Studie folgende Datenlage (vgl. dazu auch die Übersicht in Tabelle 1): Es wurden insgesamt acht Schüler/innen im Alter von 10 bis 13 Jahren interviewt (fünf Mädchen und drei Jungen). An jeder der drei Gruppendiskussionen haben jeweils fünf bis sechs Schüler/innen im Alter von 10 bis 15 Jahren teilgenommen (in allen waren sowohl Mädchen als auch Jungen vertreten). Die interviewte Lehrkraft ist weiblich und war zum Datum des ersten Interviews 54 Jahre alt, die interviewte Person des weiteren pädagogisch tätigen Personals ist ebenfalls weiblich und war 52 Jahre alt. Bei den teilnehmenden Beobachtungen im Angebot zur Aufgabenbearbeitung waren 12 bis 16 Schüler/innen und jeweils eine Angebotsleitung anwesend.

In Interviews und Gruppendiskussionen wird vom pädagogischen Personal und den Schüler/innen auch die Zeit vor Einführung der neuen (Haus-)Aufgabenpraktiken thematisiert. Das ermöglicht die Implementationsschritte an der Pestalozzischule anhand des umfangreichen Datenmaterials (vgl. zur Übersicht Tabelle 1) nachzuvollziehen.

Tabelle 1 Übersicht über die in die Fallrekonstruktion einbezogenen Daten

\begin{tabular}{lll}
\hline Feldphase 1 (2013) & Feldphase 2 (2014) & Feldphase 3 (2015) \\
\hline Interviews Schüler/innen & & \\
S001_1 & S001_2 & S001_3 \\
S002_1 & S002_2 & S002_3 \\
S003_1 & - & - \\
S004_1 & S004_2 & S004_3 \\
S005_1 & S005_2 & - \\
S006_1 & S006_2 & - \\
- & - & S007_3 \\
- & - & S008_3 \\
Gruppendiskussionen Schüler/innen & & \\
GD1_1 & - & GD3_3 \\
GD2_1 & - & - \\
Teilnehmende Beobachtungen & & \\
TB001_1 & TB002_2 & TB003_3a \\
- & - & TB003_3b \\
Interviews päd. Tätige & & \\
L001_1 & & L001_3 \\
- & L001_2 & P001_3
\end{tabular}

Legende: $\mathrm{S}=$ Schüler/in, $\mathrm{GD}=$ Gruppendiskussion, $\mathrm{TB}=$ Teilnehmende Beobachtung, $\mathrm{L}=$ Lehrkraft, $\mathrm{P}=$ weiteres päd. tätiges Personal; Schüler S001 ist bspw. ein Panelfall und wurde zu allen drei Feldphasen interviewt; das Interview zur Feldphase 1 wird mit „S001_1“, das zur Feldphase 2 mit „S001_2“ und das zum Feldphase 3 mit „S001_3“ bezeichnet.

3.2 Methoden der Datenerhebung und Datenanalyse

Die Interviews, Gruppendiskussionen und teilnehmenden Beobachtungen wurden mit Blick auf die Forschungsfrage von StEG-Q von geschulten Personen durchgeführt. All diese Methoden wurden in drei Feldphasen mit halbjährlichem Abstand eingesetzt, sodass sich das Potenzial für eine multiperspektivische, längsschnittliche Fallstudie ergibt. Die Interviewdurchführung ist angelehnt an Witzel und Reiter (2012) und in Kielblock und Lange

\footnotetext{
${ }^{2} \mathrm{Zu}$ jeder der drei Feldphasen wurden Interviews, Gruppendiskussionen und Beobachtungen durchgeführt.
} 
(2013) konkretisiert. Im Kern handelt es sich um ein alltagsnahes Gespräch zwischen Interviewer und Interviewtem, das stark von dem, was der Interviewte selbst für relevant zu erzählen hält, geleitet wird. Eine vergleichbare Herangehensweise wurde bei den Gruppendiskussionen mit den Schüler/innen gewählt. Die Interviews dauerten zwischen 10 und 20 Minuten, die Gruppendiskussionen zwischen 10 und 25 Minuten. Für die teilnehmenden Beobachtungen besuchten geschulte Personen die Hausaufgaben- und Lernzeiten und fixierten ihre Beobachtungen mithilfe teilstrukturierter Protokolle. Speziell ging es in den offenen Beobachtungen darum, den Ablauf und die sozialen Interaktionen zu beobachten und Notizen dazu anzufertigen (vgl. Lamnek 2010, S. 500ff.).

Die Analyse der Daten umfasst mehrere Schritte. Im Anschluss an die Auswahl relevanter Passagen anhand thematischer Segmentierungen wurde mittels In-Vivo Coding (vgl. Saldaña 2013, 91ff.) das ausgewählte Material , aufgebrochen' und anschließend entlang der Zeitachse zu einer längsschnittlichen Fallstudie verdichtet. Die Vorgehensweise zu ,single-case studies' mit längsschnittlicher Zielsetzung orientierte sich an Yin (2009). Entsprechend der Empfehlungen von Yin werden zur Analyse und (Re-)Konstruktion der Fallstudie alle vorliegenden Daten herangezogen und die Informationen aus den unterschiedlichen Datenquellen (Interviews, Gruppendiskussionen und Beobachtungen) im Sinne einer Triangulierung miteinander abgeglichen. Bei der Darstellung der Ergebnisse im folgenden Abschnitt werden an geeigneten Stellen ausgewählte Originalzitate angeführt (vgl. Yin 2009).

\section{Ergebnisse}

Im Laufe der iterativen Analysen stellten sich drei Ebenen heraus, auf denen relevante Entwicklungen der Implementation rekonstruiert werden konnten. An diesen Implementationsebenen orientiert sich die folgende Ergebnisdarstellung (zur Übersicht vgl. Abb. 1). Das sind

1. die Zeitstruktur des Angebots (Zeitstruktur)

2. das zur Durchführung des Angebots eingesetzte Personal (Personaleinsatz) und

3. die Begleitung der Lernenden durch das Personal (Lernbegleitung).

Abb. 1 Darstellung des Implementationsprozesses. Neuerungen und nötig werdende Nachsteuerung

Zeitstruktur
Personaleinsatz

Lernbegleitung

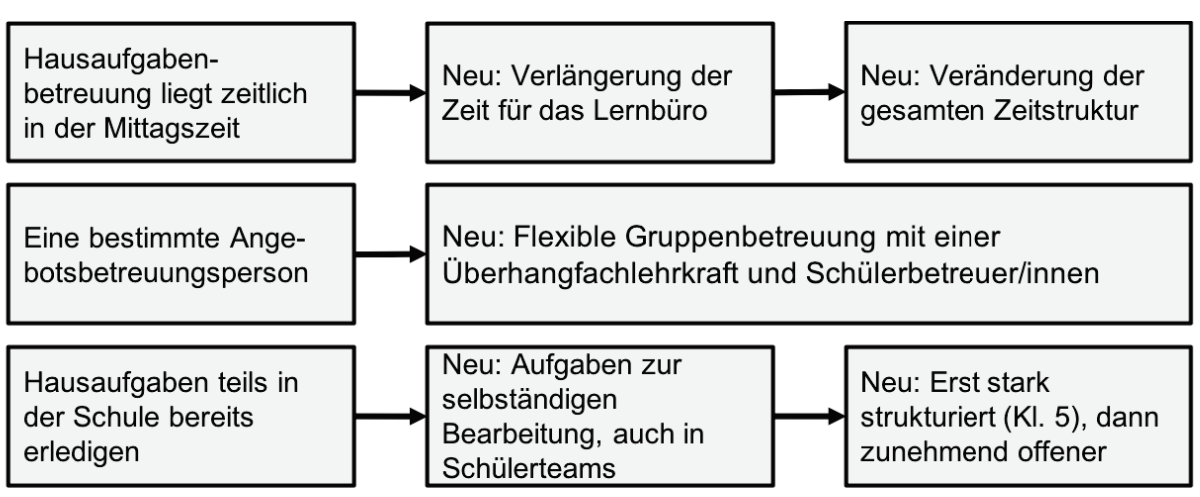

\begin{tabular}{|l|lc|}
\hline Hausaufgabenbetreuung & & Lernbüro \\
\cline { 2 - 3 } & & \\
\hline Schuljahr & Schuljahr & Schuljahr \\
$2012 / 13$ & $2013 / 14$ & $2014 / 15$ \\
\hline
\end{tabular}

\subsection{Zeitstruktur}

In den ersten beiden Schuljahren mit Ganztagsbetrieb (2011/12 und 2012/13) wurde eine reguläre Hausaufgabenbetreuung angeboten. L001_1 erklärt, dass das jetzige ,Lernbüro' früher eine Hausaufgabenbetreuung war. Es handelte sich um ein Angebot, in dem die teilnehmenden Schüler/innen die Aufgaben, die sie für zu Hause aufbekamen, bereits in der Schule erledigen konnten und dabei von einer Angebotsleitung betreut wurden. Die Hausaufgabenbetreuung wurde vor dem Mittagessen angeboten.

Zum Schuljahr 2013/14 wird die Hausaufgabenbetreuung im Zuge der schrittweisen Einführung des gebundenen Ganztags verändert. An zwei Tagen in der Woche nehmen die Schüler/innen (der Klassen 5 bis 7) verpflichtend am neu eingeführten, sogenannten ,Lernbüro' teil. L001_1 berichtet, dass die Hausaufgabenbetreuung sich ,jetzt dazu entwickeln [soll], dass wir Wochenaufgaben bearbeiten lassen“. Die Implementation einer neuen Aufgabenkultur mit einer selbstbestimmteren Bearbeitung ist damit initiiert. 
Die Betreuungszeit wird mit Einführung des Lernbüros verlängert. Nach dem Unterricht am Vormittag findet das Lernbüro nun von 11:30 bis 12:45 (75 Minuten) statt. Daran schließt sich die Mittagspause von 12:45 bis 13:30 (45 Minuten) an und schließlich gibt es einen weiteren Unterrichtsblock von 13:30 bis 15:00 (90 Minuten).

Die Verlängerung der Lernbürozeit zum Schuljahr 2013/14 wird von den Schüler/innen in zweifacher Hinsicht als problematisch wahrgenommen: Erstens steht das Lernbüro in Konkurrenz zum Mittagessen. So kritisiert S001_1: „Also das Lernbüro wurde ja jetzt verlängert. Und die Mittagspause gekürzt. Und das finden wir halt nicht so gut". Auch in einer Gruppendiskussion (GD2_1) kritisiert eine Schülerin diese Veränderung: „Das war eigentlich gut, die Zeit für das Mittagessen. Aber jetzt wurde das halt für das Lernbüro abgezogen“. Und zweitens steht das Lernbüro in Konkurrenz zu den wählbaren AGs. Hier kommentiert S001_1: „Also letztes Halbjahr war es so, dass man zwei AGs hatte. Und dadurch, dass die Lernbürozeit jetzt verlängert wurde, hat man halt nur noch eine“. Die Schüler/innen sehen diese Verlängerung der Lernbürozeit als Einschränkung ihrer Zeit für andere Aktivitäten.

Wie L001_1 zu Beginn der Einführung des Lernbüros betont, gibt es „,inzwischen fast ausschließlich Blockstunden oder Doppelstunden. Das heißt also, immer neunzig Minuten, danach immer dreißig Minuten Pausen“. Lediglich das Lernbüro fällt mit 75 Minuten und einer anschließenden Pause von 45 Minuten für das Mittagessen aus dem Takt. Damit ist ein Problem verbunden, dass das Lernbüro mit 75 Minuten nicht flexibel im Tagesablauf verschiebbar ist. Das heißt, dass eine wirkliche Rhythmisierung - in dem Sinne, das Lernbüro auch nachmittags, oder vormittags stattfinden zu lassen - nicht ohne weiteres möglich ist. Die Konkurrenz zum Mittagessen beispielsweise bleibt im bisherigen Tagesablauf unvermeidlich.

Am Ende des Schuljahrs 2013/14 gibt P001_2 daher den Ausblick, dass sich dies voraussichtlich zum nächsten Schuljahr 2014/15 ändern wird. Eine größere Umstrukturierung des gesamten Tagesablaufs soll umgesetzt werden, da es ,im Moment bei uns noch sehr starr [ist], weil durch dieses 75-Minuten-Zeitfenster es nicht mit den anderen Zeitblöcken kompatibel ist.“ Die künftige Änderung hätte aus ihrer Sicht folgenden positiven Effekt: „Damit können wir dieses Lernbüro aber nicht nur in dieser einen Schiene fahren, sondern wir können es überall hinlegen“". P001_2 betont zudem das Potenzial des Lernbüro-Zeitfensters als Nachmittagstermin. Man könnte „gucken, wo macht es Sinn, vielleicht diese Lernbüro- und AG-Zeit zu legen, die ist dann nicht immer nur kurz vor Mittag, die könnte theoretisch am Nachmittag sein“.

Die Zeitstruktur wird im folgenden Schuljahr tatsächlich verändert. Die Kürzung der Mittagspause auf 45 Minuten im Schuljahr 2014/15 wird wieder rückgängig gemacht. Sie beträgt jetzt wieder 60 Minuten.

Zudem wird das Lernbüro nun ein Mal in der Woche im letzten Block am Nachmittag zweistündig (90 Minuten) angeboten. Die Schüler/innen sehen dies kritisch. S008_3 kritisiert, dass an diesem Tag das Lernbüro in den letzten beiden Stunden zu spät ist, weil: ,[...] am Ende ist man nicht so konzentriert wie in der Mitte“.

Für die individuellen Lerntempi und die unterschiedlichen Konzentrationsphasen der Schüler/innen scheint das Konzept also nach wie vor nicht flexibel genug zu sein. S002_3 macht deutlich, dass es zwar gut ist, dass die Mittagspause mittlerweile wieder länger ist, ihr allerdings die Zeit, um die Aufgaben wirklich ordentlich zu bearbeiten, oftmals nicht ausreicht, wenn sie viele Aufgaben zu erledigen hat. Sie kommentiert: „es wurde halt diese Viertelstunde vom Lernbüro abgezogen. Das ist wiederum ein bisschen schade, weil ich halt nicht alles schaffe, was ich mir vorgenommen habe [...] und früher hatten wir halt die Viertelstunde dann noch länger und die hat man - also zumindest ich - schon gebraucht. Aber Mittagspause ist halt auch gut, wenn man die jetzt hat“.

Die vielen Umstrukturierungen hinsichtlich der zeitlichen Gestaltung des Lernbüros werden von den Beteiligten als anstrengend wahrgenommen. Zu Anfang des Schuljahrs 2014/15 berichtet L001_3 von „ständigen Veränderungen“ und dass ,immer noch mal wieder rumgefeilt werden muss“ am Ganztagsbetrieb - so auch am Lernbüro. S002_2 bestätigt, dass es bei der Ausgestaltung des Lernbüros zwar Unstimmigkeiten gibt, es aber toll ist, seine Meinung äußern und an der Entwicklung des Lernbüros mitarbeiten zu dürfen. Aus Sicht von L001_3 müsste es so sein, „dass man eigentlich von Anfang an ein Gesamtkonzept braucht“.

\subsection{Personal}

Vor Einführung des Lernbüros war für die Hausaufgabenbetreuung eine Person des pädagogischen Personals zuständig. Mit Einführung des neuen Konzepts findet das Lernbüro für alle Klassen einer Jahrgangsstufe an zwei festen Tagen in der Woche im Klassenverband statt. An diesen Tagen übernimmt jeweils die Klassenlehrkraft die Leitung des Lernbüros. An einem dritten Tag werden jeweils zwei Klassen aus einem Jahrgang für das Lernbüro zusammengefasst, da ein Teil der Schüler/innen parallel dazu in einer Wahl-AG ist. Eine Schülerin merkt dazu an, dass ihr das Lernbüro sehr gut gefällt, wenn die eigene Klassenlehrkraft das Lernbüro leitet. Wenn jedoch andere Lehrkräfte die Leitung übernehmen, sind die Schüler/innen ,alle immer so laut“ (S006_2). 
Um eine für die Schüler/innen bedarfsgerechte Begleitung bei der Bearbeitung der Aufgaben zu ermöglichen, wird mit der Einführung des Lernbüros angestrebt, dass zur Lernbürozeit stets Fachlehrer/innen aus den Hauptfächern für Fragen und Hilfestellungen verfügbar sind. „Und dann haben wir also, wenn es geht, einen Deutsch-, Englisch, Mathelehrer vor Ort", sagt L001_1. Ein halbes Jahr später scheint sich diese Praktik gefestigt zu haben und wird von den Schüler/innen angenommen: ,unsere Mathelehrerin ist auch Donnerstags im Lernbüro und dann können wir sie halt auch noch fragen“ (S001_2). Zwischen den verschiedenen Fachlehrerkräften und der Klassenleitung entwickelt sich außerdem eine Kooperation bzw. ein regelmäßiger Austausch zum aktuellen Lernstand der Schüler/innen. So erklärt L001_2 im Interview: „Ja, es sind ja nicht immer die Klassenlehrer im Lernbüro, sondern auch andere, und es sind zusätzlich dann immer Fachlehrer noch anwesend, so dass man die Schüler auch einfach mal dahin schicken kann bei einer speziellen Frage und sagen: ,Frag mal', oder ,geh mit deinem Problem dort hin'. Ja, klar, das müsste wahrscheinlich noch ein bisschen intensiviert werden, die Zusammenarbeit, aber ganz ohne läuft das einfach nicht. Der Fachlehrer muss ja, wenn er im Lernbüro ist, wissen, was ist gelaufen“.

Aus der Sicht der Schüler/innen ist die Betreuung durch die Lehrkräfte im Lernbüro hilfreich. Sie bekommen von den Lehrkräften Tipps, die sie zu Hause nicht bekämen, sagt eine Schülerin in einer Gruppendiskussion (GD2_1): „Weil die Lehrer helfen mir besser als meine Eltern, sage ich mal. Weil die wissen ja auch, wo wir Hilfe brauchen, nicht? Und in der Grundschule war es so, ich habe mich immer mit den Hausaufgaben ewig lange herumgeschlagen. [...] Meistens bis Fünf oder so, je nachdem, was wir aufhatten. Und jetzt habe ich das in meinen 45, nee, 75 Minuten fertig“.

Unterstützt werden die Schüler/innen der 5. und 6. Klassen bei der selbständigen Bearbeitung ihrer Aufgaben im Lernbüro nicht nur von der anwesenden Lehrkraft, sondern an zwei Tagen in der Woche auch von Schüler/innen aus höheren Klassen. Es gibt „Lernhelfer“ aus den 9. und 10. Klassen, erklärt P001_2, die Schulleitung, im zweiten Halbjahr nach Einführung des Lernbüros. Sie „,unterstützen Schüler quasi zu zweit oder zu dritt neben dem Lehrer noch beim Bearbeiten ihrer Aufgaben, Übungsaufgaben und so weiter und so fort. [...] Und die Schüler machen das sehr gern, also die Großen sind da echt heiß drauf, auf den Job“.

Von S002_1 wird berichtet, dass die Schülerbetreuer aus den höheren Klassen manchmal „ein bisschen albern [sind] und dann lenkt das ziemlich ab von den Sachen, die wir machen müssen" und gelegentlich wissen sie auch nicht, wie die Aufgaben zu lösen sind. Ein halbes Jahr später berichtet die Schülerin davon, dass die Probleme mit den Schülerbetreuern mittlerweile besprochen wurden und das Ganze jetzt besser ablaufen würde. Im darauffolgenden Schuljahr - mittlerweile ist die Schülerin in der 7. Klasse, in der keine Schülerbetreuer mehr zur Unterstützung im Lernbüro vorgesehen sind - sagt sie, dass ihr die Schülerbetreuer im Lernbüro sogar fehlen. Die betreuende Lehrkraft im Lernbüro ist nämlich nicht jederzeit für Fragen ansprechbar und so war es hilfreich sich mit Problemen auch an die Lernbegleiter wenden zu können. Auch in einer Gruppendiskussion kommt zur Sprache, dass die Schülerbetreuer in manchen Fällen sogar besser erklären können als die Lehrkräfte (GD1_1).

\subsection{Lernbegleitung}

Am Ende des ersten Schuljahrs mit neu eingeführtem Lernbüro (2013/14) stellt L001_2 fest, dass sich damit „die Aufgaben dann natürlich wandeln müssen. [...] Jetzt wollen wir selbstständiges Arbeiten ja erreichen, und die kriegen dann also eher Aufgaben, die sie über einen längeren Zeitraum erfüllen müssen oder auch Aufgaben, die sie mit Partner oder in Gruppenarbeit erledigen müssen“". Vom Wandel ist also nicht bloß das Angebot ,Lernbüro“ selbst betroffen. Es müssen zudem die Aufgabenpraktiken in den Fächern verändert, die neue Aufgabenkultur etabliert und schließlich von den Schüler/innen angenommen werden.

Mit der Einführung des Lernbüros zum Schuljahr 2013/14 ist die zentrale Neuerung in der pädagogischen Konzeption der Hausaufgabenpraxis, dass die Schüler/innen zunehmend selbständig an ihren Aufgaben arbeiten sollen. Verschiedene Arbeitsweisen werden zum Erreichen dieses Ziels eingeführt. Das Lernbüro wird zum Beispiel in zwei Phasen eingeteilt. In der ersten Phase geht es darum, dass die Schüler/innen für sich noch einmal sichten, „was habe ich noch zu tun, welche Aufgaben muss ich erledigen heute, verstehe ich meine Aufgaben, wo habe ich Fragen“ (L001_1). Dann gibt es ,anschließend Gelegenheit nochmal zu fragen, wenn Unklarheiten sind“ (L001_1). In der zweiten Phase gehen die Schüler/innen dann an ihre Aufgaben, bei denen kooperative Arbeitsweisen der Schüler/innen ausdrücklich erlaubt und erwünscht sind. Wenn weitere Fragen auftauchen oder alles erledigt ist, kommen die Schüler/innen zu den Lehrkräften, „,sodass man sich nochmal beraten kann [...]. Ja, ganz zum Schluss soll dann nochmal überprüft werden, ob alles erledigt wurde, was zu erledigen ist“"(L001_1).

Dass sich die geplante, selbstbestimmte und selbstorganisierte Arbeit an den Wochenaufgaben nicht ohne weiteres implementieren lässt, zeigt die Passage aus einem Interview mit L001_2: „Wir haben gemerkt, es muss ein wenig Struktur haben, vor allen Dingen in der Fünf und Sechs [gemeint sind die Jahrgangsstufen 5 und 6, Anm. d. Verf.], 
[...] wir hatten das am Anfang auch offener geplant und mussten so ein bisschen zurück rudern“. Die Lehrkraft verdeutlicht im Interview, dass die jüngeren Schüler/innen noch nicht in der Lage sind ihre anstehenden Aufgaben gänzlich selbst zu strukturieren. Durch klarere Anweisungen seitens der Angebotsleitungen, was wann zu tun ist, gelingt es den Schüler/innen besser ihre Aufgabenbearbeitung am Anfang des Lernbüros zu planen.

Die Lehrkräfte strukturieren die Arbeit mit den Wochenaufgaben zu Beginn der 5. Klasse sehr stark. Auf diese Weise sollen sich die Schüler/innen nach und nach an die selbstorganisierte Arbeitsweise gewöhnen. „Es ist zunächst sehr strikt, die ersten Wochen, damit sie einfach merken, wie lange dauern etwa zwanzig Minuten, denn die habe ich pro Fach, und dann wechseln wir" (L001_2). Um die Erledigung der Aufgaben besser organisieren und planen zu können, bekommen die Schüler/innen ein Hilfsmittel an die Hand: Sie haben einen Schulplaner, ,in dem sie ihre Aufgaben notieren, und sie können dann schon selbstständig entscheiden, welche Aufgaben gehe ich zuerst an, welche hebe ich mir auf. Oder habe ich mich vielleicht schon vorher mit anderen verabredet, dass wir sagen, die erste halbe Stunde arbeitet jeder für sich, und dann treffen wir uns, denn häufig machen wir das ja auf den Fluren, arbeiten wir zusammen an einem Thema“"(L001_2).

\section{Diskussion}

Der vorliegende Beitrag widmete sich der Frage, wie sich die Umstellung von einer konventionellen Hausaufgabenpraxis (Hausaufgaben + Hausaufgabenbetreuung) zu einer integrierten Lernzeit vollzieht. Untersucht wurde anhand der Einzelfallstudie, zu welchen Veränderungen es auf den Ebenen der Implementation kommt (Zeitstruktur, Personaleinsatz und Lernbegleitung) und wo im Laufe der ersten Schulhalbjahre nach Einführung der Lernzeit nachgesteuert werden musste.

\section{Zeitstruktur}

Die Verlängerung der Zeit, die für das Lernbüro vorgesehen ist, wird von den Schüler/innen mit Kritik aufgenommen: Die Schüler/innen zeigen sich reflektiert, was ihr Recht auf eine ausreichend lange Mittagspause sowie die Zeit für Wahl-AGs angeht. Dass sie im dritten Halbjahr nach Einführung der Lernzeit, als die Mittagspause wieder ihre ursprüngliche Länge hat, sich gehört fühlen und mit der nun bestehenden Zeitstruktur zufrieden sind, zeigt sich deutlich in den Interviews. Dies deutet an, dass neue Praktiken nicht ohne die Nachvollziehbarkeit bei den Schüler/innen eingeführt werden sollten. Dass die Umstrukturierung der Zeitstruktur des gesamten Schultags erst nach Einführung der neuen Aufgabenpraktiken erfolgt ist, hätte durch ein besseres Gesamtkonzept von Beginn der Einführung des Ganztags besser gelöst werden können.

Eine zu starre Einbettung der Lernbürozeit wird den Schülerbedürfnissen nicht gerecht. An der Schule wurde das Problem erkannt und mit dem Ziel, eine umfassende Tagesrhythmisierung einzuführen, bearbeitet. Es zeigt sich, dass eine neue Zeitstruktur nicht ohne weiteres umgesetzt werden kann. Durch neue Rhythmisierung des Schultags wird das Lernbüro-Zeitfenster flexibel im Tagesverlauf verschiebbar.

\section{Personaleinsatz}

Mit Einführung des Lernbüros und der verpflichtenden Teilnahme für die Schüler/innen wird für die Durchführung des Angebots mehr Personal benötigt. Während, früher' bei der Hausaufgabenbetreuung nur eine pädagogische Fachkraft zur Beaufsichtigung der Schüler/innen nötig war, findet das Lernbüro an zwei Tagen in der Woche im Klassenverband mit der jeweiligen Klassenlehrkraft statt. Das Bearbeiten der Wochenaufgaben in der gewohnten Lerngruppe und mit der gewohnten Lehrkraft lässt sich direkt erfolgreich umsetzen. Die Schüler/innen berichten in den Interviews nicht nur, dass sie die Betreuung durch die Lehrkräfte häufig als hilfreicher ansehen als bei der Erledigung zu Hause, sondern auch, dass sie in der Schule ihre Zeit im Lernbüro effektiver nutzen. Um die fachliche Unterstützung der Kinder zu verbessern, ist an einem Tag in der Woche jeweils zusätzlich eine Fachlehrkraft zu den Lernbürozeiten anwesend, die den Schüler/innen fachspezifische Hilfe anbietet. Zwischen den durchführenden Lehrkräften entwickelt sich im Zuge dieser neuen Aufgabenteilung eine Kooperation (Absprachen etc.). Zusätzlich werden Schüler/innen aus höheren Klassenstufen an einem Tag pro Woche als Lernbegleiter für die Jahrgänge 5 und 6 eingesetzt. Das funktioniert nicht von Anfang an reibungslos, spielt sich im Laufe des ersten Schuljahrs jedoch ein.

\section{Lernbegleitung}

Die Fallstudie zeigt, dass die Implementation von selbstbestimmten (Haus-)Aufgabenpraktiken (hier mit Wochenplan) nicht ad hoc für alle Klassenstufen funktioniert. Gerade die Schüler/innen aus niedrigeren Klassenstufen brauchen - sofern das Konzept für sie neu ist - zu Beginn deutlich mehr Struktur als es von den beteiligten Lehrkräften zunächst erwartet wurde. Ein schrittweises Einführen der neuen Praktiken erleichtert die Umsetzung für 
alle Beteiligten. Zum einen wird die Lernbürozeit in zwei Phasen (,Stillarbeitsphase“ und anschließend eine Phase mit zunehmend kooperativen Lernformen) geteilt, um den Schüler/innen mehr Struktur zu bieten. In Jahrgangsstufe 5 geben die Lehrkräfte außerdem konkrete Zeitfenster für die Bearbeitung fachbezogener Aufgaben, um die Schüler/innen in ihrem Zeitmanagement zu unterstützen.

\section{Limitationen der vorliegenden Studie}

Die methodische Anlage des gesamten Projekts war darauf gerichtet, ein eher breites Bild der außerunterrichtlichen Angebote zu generieren. Möglichkeiten der vertieften, längsschnittlichen Analyse bestimmter Angebote, wie es in der vorliegenden Studie umgesetzt wurde, ergaben sich im Projektkontext daher mehr oder weniger zufällig aus den Gegebenheiten an dem jeweiligen Schulstandort. Entsprechend könnte ein Projekt, das speziell die organisatorischen Übergänge von Hausaufgaben in Lernzeiten an Ganztagsschulen fokussiert betrachtet, zielgerichteter Daten erheben und könnte daher deutlich facettenreicher die entstehenden Dynamiken abbilden. In der vorliegenden Studie konnten einige Grundsteine für solche vertieften Forschungsvorhaben gelegt werden, die es in künftigen Studien durchzuführen gilt. Ein weiterer kritischer Punkt betrifft die Möglichkeit die Komplexität des Falls in geeigneter Form darzustellen. Zwar wurde versucht, das empirische Material hinter den Analysen immer auch als aussagekräftige Belege an einigen Stellen anzuführen - jedoch konnte nur ein Bruchteil des analysierten Materials in dieser Weise sichtbar gemacht werden.

\section{Fazit}

Die Integration der Hausaufgaben in den Schultag kann zur Erfüllung des Anspruchs an die Ganztagsschule beitragen, eine neue Lehr-und Lernkultur zu schaffen. Die längere Zeit, die die Schüler/innen in der Schule verbringen, muss zu einem veränderten Verständnis vom Lernen und vom aktiv handelnden Kind führen (Buchner 2012, S. 120-121), wenn Ganztagsschule nicht einfach nur eine Verlängerung des Unterrichts plus Betreuung als ,Verwahrung' der Kinder werden soll.

Dieser Beitrag leistet zur Schul- und Ganztagsschulforschung einen wichtigen Beitrag. Die ablaufenden Prozesse bei der Implementation selbstverantwortlicher und selbstgesteuerter Hausaufgaben- und Lernangebote können anhand der beschriebenen Fallstudie sowie unter Einbezug der Zeitstruktur, des Personals und der Lernbegleitung besser verstanden werden. Obwohl unterschiedliche Schulstandorte sehr individuelle Grundvoraussetzungen und Dynamiken aufweisen, dürften die in dieser Studie beschriebenen fundamentalen Ebenen für nahezu alle Schulen, die sich auf den Weg zum Abbau der Hausaufgaben machen wollen, mehr oder weniger aufwändig zu bewältigen sein.

Ein besonders für die Ganztagsschul- und Unterrichtsentwicklung bemerkenswerter Aspekt der Fallstudie ist, dass die Strategie gelungen scheint die Beteiligten - auch die Schüler/innen - in die Weiterentwicklung der Gestaltung miteinzubeziehen. Sie werden nach ihrer Meinung gefragt und fühlen sich, was ihre Belange in der Schule angeht, nicht handlungsunfähig. Das knüpft an aktuelle Diskussionen um die Implementation einer neuen Aufgabenkultur an Ganztagsschulen an: „Im Prozess von den Hausaufgaben zur Arbeit an Aufgaben in Lernzeiten müssen alle Beteiligten diese Bedingungen an ihrer eigenen Schule als Grundlage gemeinsam erfahren und festlegen. Im Anschluss daran können sie die Situation an ihrer Schule neu gestalten und verbessern“ (Boßhammer und Schröder 2012, S. 70). Dass sich die vielen Veränderungen und Nachsteuerungen, die bei den Beteiligten teilweise zu Unmut führten, so besser aushalten lassen und die Mitbestimmung den Atem bei Entwicklungsprozessen in der Ganztagsschule verlängert, spricht aus Sicht der Autor/innen deutlich für eine offene und diskursive Ganztagsschulentwicklung, welche die Stimmen der Beteiligten hört und in den Entwicklungsprozess aktiv einbezieht.

\section{Literaturverzeichnis}

Börner, N., Conraths, A., Gerken, U., Steinhauer, R., Stötzel, J. \& Tabel, A. (2014). Bildungsbericht Ganztagsschule $N R W$ 2014. Institut für soziale Arbeit e. V.

Börner, N., Gerken, U., Stötzel, J. \& Tabel, A. (2013). Bildungsbericht Ganztagsschule NRW 2013. Institut für soziale Arbeit e. V.

Boßhammer, H. \& Schröder, B. (2012). Von den Hausaufgaben zu Aufgaben in der Ganztagsschule. In S. Appel \& U. Rother (Hrsg.), Schulatmosphäre - Lernlandschaft - Lebenswelt (Jahrbuch Ganztagsschule, Bd. 2012, S. 67-81). Schwalbach: Wochenschau.

Buchner, T. (2012). Von Hausaufgaben zu Lernzeiten. Südschule Lemgo 2005-2011. In S. Appel \& U. Rother (Hrsg.), Schulatmosphäre - Lernlandschaft - Lebenswelt (Jahrbuch Ganztagsschule, Bd. 2012, S. 112-121). Schwalbach: Wochenschau.

Dieckmann, K., Höhmann, K. \& Tillmann, K. (2008). Schulorganisation, Organisationskultur und Schulklima an ganztägigen Schulen. In H. G. Holtappels, E. Klieme, T. Rauschenbach \& L. Stecher (Hrsg.), Ganztagsschule 
in Deutschland. Ergebnisse der Ausgangserhebung der ,, Studie zur Entwicklung von Ganztagsschulen “ (StEG) (2. Aufl., S. 164-185). Weinheim: Juventa.

Dollinger, S. (2014). Ganztagsschule neu gestalten. Bausteine für die Schulpraxis. Weinheim: Beltz.

Emden, M. (2016). Lern- statt Hausaufgaben. Chemielernen im Ganztag. In N. Fischer, H. P. Kuhn \& C. Tillack (Hrsg.), Was sind gute Schulen? Teil 4: Theorie, Praxis und Forschung zur Qualität von Ganztagsschulen (Theorie und Praxis der Schulpädagogik, Band 38, S. 188-200). Immenhausen: Prolog.

Gaiser, J. M., Kielblock, S. \& Stecher, L. (2016). Hausaufgabenangebote an Ganztagsschulen. Fallstudien zur Verzahnung von Unterricht und außerunterrichtlichen Angeboten. Zeitschrift für Pädagogik, 62 (6), 797-811.

Grimm, W. \& Schulz-Gade, G. (2015). Übungs- und Lernzeiten an der Ganztagsschule. Ein Praxisleitfaden zur Integration von Hausaufgaben in den Ganztag. Schwalbach: Debus Pädagogik.

Haenisch, H. (2009). Verzahnung zwischen Unterricht und außerunterrichtlichen Angeboten im offenen Ganztag. Eine qualitative Studie zu praktischen Ansätzen der Verzahnung in ausgewählten Schulen. Der GanzTag in NRW-Beiträge zur Qualitätsentwicklung, 5 (11).

Hascher, T. \& Bischof, F. (2000). Integrierte und traditionelle Hausaufgaben in der Primarschule - ein Vergleich bezüglich Leistung, Belastung und Einstellungen zur Schule. Psychologie in Erziehung und Unterricht, 47 (4), 252-265.

Hessisches Kultusministerium. (2018). Richtlinie für ganztägig arbeitende Schulen in Hessen nach $\S 15$ Hessischen Schulgesetz. Zugriff am 06.12.2018. Verfügbar unter www.rv.hessenrecht.hessen.de

Heyl, K., Fischer, N. \& Tillack, C. (2016). Annahmen und Befunde zur Hausaufgabenbetreuung in Ganztagsschulen. In N. Fischer, H. P. Kuhn \& C. Tillack (Hrsg.), Was sind gute Schulen? Teil 4: Theorie, Praxis und Forschung zur Qualität von Ganztagsschulen (Theorie und Praxis der Schulpädagogik, Band 38, S. 177-187). Immenhausen: Prolog.

Heyl, K., Gaiser, J. M., Kielblock, S. \& Fischer, N. (2018). Hausaufgabenbetreuung in der Ganztagsschule - Potenziale für individuelle Förderung. Diskurs Kindheits- und Jugendforschung, 13 (3), 361-367.

Höhmann, K. (2005). Hausaufgaben als pädagogische und organisatorische Herausforderung. In K. Höhmann, H. G. Holtappels, I. Kamski \& T. Schnetzer (Hrsg.), Entwicklung und Organisation von Ganztagsschulen. Anregungen, Konzepte, Praxisbeispiele (S. 77-83). Dortmund: IFS.

Kaufmann, E. (2013). Ganztag ohne Hausaufgaben!? Forschungsergebnisse zur Gestaltung von Übungs- und Lernzeiten. Stiftung Universität Hildesheim.

Kielblock, S. (2015). Program Implementation and Effectiveness of Extracurricular Activities. An Investigation of Differential Student Perceptions in Two German All-Day Schools. International Journal for Research on Extended Education, 3 (2), 79-98.

Kielblock, S. \& Lange, A. (2013). Das problemzentrierte Interview. Grundlagen und Forschungspraxis. Zeitschrift für Soziologie der Erziehung und Sozialisation, 33 (4), 439-448.

Lamnek, S. (2010). Qualitative Sozialforschung (5. Aufl.). Weinheim: Beltz.

Meyer-Hamme, A. (2014). Außerunterrichtliche Aktivitäten und herkunftsbedingte Diversität. Konzeption und Wahrnehmung an Ganztagsschulen. Weinheim: Beltz Juventa.

Nordt, G. (2013). Lernen und Fördern in der Hausaufgabenpraxis der offenen Ganztagsgrundschule in NordrheinWestfalen. Eine qualitative Studie aus der Perspektive der pädagogischen Kräfte und der Kinder. Münster: Waxmann.

Nordt, G. \& Röhner, C. (2008). Hausaufgaben in der offenen Ganztagsgrundschule - ein Beitrag zur Förderung des schulischen Lernens und der Schulqualität? Widersprüche, 28 (110), 67-79.

Rabenstein, K. \& Podubrin, E. (2015). Praktiken individueller Zuwendung in Hausaufgaben und Förderangeboten. Empirische Rekonstruktionen pädagogischer Ordnungen. In S. Reh, B. Fritzsche, T.-S. Idel \& K. Rabenstein (Hrsg.), Lernkulturen. Rekonstruktion pädagogischer Praktiken an Ganztagsschulen (S. 219-263). Wiesbaden: VS.

Radisch, F., Stecher, L., Klieme, E. \& Kühnbach, O. (2008). Unterrichts- und Angebotsqualität aus Schülersicht. In H. G. Holtappels, E. Klieme, T. Rauschenbach \& L. Stecher (Hrsg.), Ganztagsschule in Deutschland. Ergebnisse der Ausgangserhebung der „, Studie zur Entwicklung von Ganztagsschulen “ (StEG) (2. Aufl., S. $227-$ 260). Weinheim: Juventa.

Rollett, W. \& Tillmann, K. (2009). Personaleinsatz an Ganztagsschulen. In I. Kamski, H. G. Holtappels \& T. Schnetzer (Hrsg.), Qualität von Ganztagsschule. Konzepte und Orientierungen für die Praxis (S. 132-143). Münster: Waxmann.

Saldaña, J. (2013). The coding manual for qualitative researchers (2. Aufl.). Thousand Oaks: SAGE. Schulgesetz Berlin (2016). http://www.schulgesetz-berlin.de/berlin/schulgesetz.php (Zugriff: 22.3.2018)

Standop, J. (2013). Hausaufgaben in der Schule. Theorie, Forschung, didaktische Konsequenzen. Bad Heilbrunn: Julius Klinkhardt. 
StEG Konsortium. (2016). Ganztagsschule: Bildungsqualität und Wirkungen außerunterrichtlicher Angebote. Ergebnisse der Studie zur Entwicklung von Ganztagsschulen 2012-2015. Frankfurt am Main.

StEG-Konsortium. (2015). Ganztagsschule 2014/2015. Deskriptive Befunde einer bundesweiten Befragung. Frankfurt am Main.

Voag, A. (2006). Anregungen zur Veränderung der Hausaufgabenpraxis am Ganztagsgymnasium. In S. Appel, H. Ludwig, U. Rother \& G. Rutz (Hrsg.), Ganztagsschule gestalten (Jahrbuch Ganztagsschule, Bd. 2007, S. 171181). Schwalbach: Wochenschau.

Wild, E. \& Lorenz, F. (2010). Elternhaus und Schule. Paderborn: Schöningh.

Witzel, A. \& Reiter, H. (2012). The problem-centred interview. Principles and practice. London: SAGE. Yin, R. K. (2009). Case study research. Design and methods (4. Aufl.). Los Angeles: SAGE. 